\title{
On the Optimal Control of a Class of Time-Delay System
}

\author{
L. Boudjenah*, M.F. Khelfi \\ Department of Computer Science, University of Oran BP 1524 Oran 31000, Algeria
}

\begin{abstract}
In this work we study the optimal control problem for a class of nonlinear time-delay systems via paratingent equation with delayed argument. We use an equivalence theorem between solutions of differential inclusions with time-delay and solutions of paratingent equations with delayed argument. We study the problem of optimal control which minimizes a certain cost function. To show the existence of optimal control, we use the main topological properties of the set solutions of paratingent equation with delayed argument.
\end{abstract}

Key words: differential inclusion, minimization, optimal control, paratingent, time-delay AMS subject classification: 34A60, 49J27, 93C10

\section{Introduction}

In the time-delay systems (called also hereditary or systems with aftereffects) the evolution of current states depends on the past values of states and/or controls. Their evolution is described by differential equations which include information on the past history. The present state of a timedelay system is determined in some way by its past history. The reaction of real world systems to exogenous signals is never instantaneously and it needs some delay. In general, a time-delay system arises as a result of inherent delays in the transmission of information between different parts of the system and/or as a deliberate introduction of time delay into the system for control purposes. Such systems exist in various fields of application, such as in physics, engineering, biology, medicine, and economics. The delay effects on control of dynamical systems are problems of recurring interest since the delay presence may induce complex behaviors (oscillations, instability). In recent years considerable interest has been shown in time-delay control systems: control, stability, etc... see Kuang [5] and references therein. The optimal control problem for nonlinear

\footnotetext{
${ }^{*}$ Corresponding author. E-mail: lotfi60@yahoo.fr
} 
time-delay systems has been receiving constant attention, see S. Dadebo and R. Luus [3] and references therein. In the mathematical framework, such systems can be described by differential inclusions. A more extended survey on differential inclusions can be found in Aubin and Cellina [1] and Kisielewicz [4].

In [2] we have established the existence of solutions to a class of paratingent equation with delayed argument under weak assumptions. In this work we study the optimal control problem for nonlinear time-delay systems under the same weak assumptions as in [2]. To show the existence of optimal control, we use an equivalent theorem between solutions of paratingent equation with delayed argument and solutions of differential inclusions with time-delay.

\subsection{Preliminaries and notations}

Let $E$ be a vector space. By CompEwe denotes the set of all the nonempty and compact subsets of $E$ and Conv $E$ denotes the set of all the convex elements of $C o m p E$. Let $C$ be the space of all continuous functions $x: R \rightarrow R^{n}$ with the topology defined by an almost uniform convergence, $\beta<0$ a fixed real number and $I=\left[0, \infty\left[\subset R\right.\right.$. If $x \in C$, the symbol $[x]_{t}$ will be denote the restriction of $x$ on the interval $[\beta, t]$ where $t \in I$ and $\|x\|_{t}=\max \{|x(s)|, \beta \leq s \leq t\}$ with $|x|=\max \left\{\left|x_{1}\right|,\left|x_{2}\right|, \ldots,\left|x_{n}\right|\right\}$ for $x=\left(x_{1}, x_{2}, \ldots, x_{n}\right) \in R^{n} . G$ denotes the metric space whose elements are functions $[x]_{t},[y]_{u}, \ldots$, where $t \in I, u \in I$, the distance between two functions $[x]_{t}$, $[y]_{u}$, being understood as a distance of their graphs in $R \times R^{n}$ in the Hausdorff sense.

Having a function $x \in C$ and $t \in I$, the set of limit points: $\lim \frac{x\left(u_{i}\right)-x\left(s_{i}\right)}{u_{i}-s_{i}}=\alpha$, where $u_{i} \in I$, $s_{i} \in I, u_{i} \neq s_{i}(i=1,2, \ldots)$, and $\lim u_{i}=\lim s_{i}=t$, is called the paratingent of $x$ at the point $t$ and denoted by $(P t x)(t)$.

Let a set-valued map $F: G \rightarrow C o m p R^{n}$, a relation of the form:

$$
(P t x)(t) \subset F\left([x]_{t}\right),
$$

is called paratingent equation with delayed argument where $t \in I$ and $x \in C$. Every function $x \in C$ satisfying (1) will be called the solution of these equation. The generalized Cauchy problem for the equation (1) consists in the search for a solution of the equation (1) which will be satisfy the initial condition:

$$
x(t)=\xi(t) \quad \text { for } \quad t \in[\beta, 0],
$$

where the function $\xi \in C$, called the initial function is given in advance.

Assume the following hypothesis: (H1) the set-valued map $F: G \rightarrow C o n v R^{n}$ is upper semicontinuous and satisfies the condition: $F\left([x]_{t}\right) \subset \bar{B}\left(0, w\left(t,\|x\|_{t}\right)\right)$ for $t \geq 0$, where $\bar{B}(0, r)$ will denote the closed ball of center 0 of $R^{n}$ and radius $r, w(t, y)$ is a continuous function from $I \times I$ to $I$, increasing in $y$ and such that the ordinary differential equation: $y^{\prime}=w(t, y)$, with the initial condition $y(0)=A$ ( $A$ an arbitrary real positive number) has a maximal solution, denoted $M(t)$, on all intervals $I$ and for all $A$.

Let $D$ be a compact subset of $C$ and $\Phi$ the class of all functions $x \in C$ satisfying the inequality $|x(t)| \leq M(T)$ for $t \in[0, T], T$ be an arbitrarily fixed real positive number. 
Definition 1. A function $x$ is called a trajectory of $F\left([y]_{t}\right)$, if $(P t x)(t) \subset F\left([x]_{t}\right)$ for $t \geq 0$. We call emission of the function $\zeta$, the set of all trajectories $x$ of $F\left([y]_{t}\right)$ such that $x \in \Phi$ and $x(t)=\zeta(t)$ for all $t \in[\beta, 0]$

In [2] we have established the following theorem.

Theorem 2. Under the hypothesis (H1), in class $\Phi$ and for any $\xi \in D$, there exist at least one solution of the problem (1.1)-(1.2).

\section{Time delay control system}

In this section we will state and prove the main result. First, we will state the equivalence theorem. Let $\beta<0$ be a fixed real number, $T$ an arbitrarily fixed positive real number, $U$ a closed set of $R^{m}$, $F$ a set-valued map: $G \times U \rightarrow \operatorname{Comp}^{n}, Q$ a set-valued map: $G \rightarrow \operatorname{Comp} R^{m}$ and $\zeta$ a definite and continuous function on $[\beta, 0]$.

Definition 3. We call time-delay control system, and we note $S(F, Q)$, a pair of set-valued map: $F\left([x]_{t}, u\right)$ and $Q\left([x]_{t}\right)$. We call trajectory or solution of the time-delay control system $S(F, Q)$ any function $x(t)$ defined and continuous on $[\beta, T]$ absolutely continuous on $[0, T]$ and such that: $x^{\prime}(t) \in F\left([x]_{t}, u\right)$ a.e on $[0, T]$ and $x(t)=\zeta(t)$ for $t \in[\beta, 0]$, where $u(t)$ is a measurable function on $[0, T]$ verifying: $u(t) \in Q\left([x]_{t}\right)$ a.e on $[0, T]$. The relation $(P t x)(t) \subset R\left([x]_{t}\right)$ for $t \in[0, T]$ and $x(t)=\zeta(t)$ for $t \in[\beta, 0]$ is called paratingent equation with delayed argument associate to the control system $S(F, Q)$ where $R\left([x]_{t}\right)=F\left([x]_{t}, Q\left([x]_{t}\right)\right)=\left\{F\left([x]_{t}, u\right) / u \in Q\left([x]_{t}\right)\right\}$.

Theorem 4. Let $\zeta$ be a defined and continuous function on [ $\beta, 0], T$ an arbitrarily fixed positive real number and $R: G \rightarrow C$ Conv $R^{n}$ a set valued map verifying the assumptions of Theorem 1 , then the following statements are equivalent: (P1) A function $x \in C$ is a solution of the paratingent equation with delayed argument: $(P t x)(t) \subset R\left([x]_{t}\right)$ for $t \in[0, T], x(t)=\zeta(t)$ for $t \in[\beta, 0]$. (P2) A function $x \in C$, absolutely continuous on $[0, T]$ is a solution of the differential inclusion with time-delay: $x^{\prime}(t) \in R\left([x]_{t}\right)$ a.e on $[0, T], x(t)=\zeta(t)$ for $t \in[\beta, 0]$.

Proof. i) It i easy to show that $(\mathrm{P} 1) \Rightarrow(\mathrm{P} 2)$ from proof of Theorem 1 , (see[2]).

ii) We will show that $(\mathrm{P} 2) \Rightarrow(\mathrm{P} 1)$. Let $x \in C$ a function verifying the proposition (P2). Let us fix $t$ in $[0, T]$. The map $R$ being upper semi-continuous on $[0, T]$, then there exists an interval $[a, b] \subset I$ containing $T$ and such that we have: $\forall s \in[a, b], R\left([x]_{t}\right) \subset B\left(R\left([x]_{s}\right), \epsilon\right)$, (see [1]). Since $x^{\prime}(s) \in R[x]_{s}$ a.e on $[a, b]$, from Lemma 2 of [6] we have: $\frac{x(\tau)-x(\sigma)}{\tau-\sigma} \in B\left(R\left([x]_{s}\right), \epsilon\right)$ for $\tau, \sigma \in[a, b]$. Passing to the limit when $\tau \rightarrow t$ and $\sigma \rightarrow t$ we obtains: $(P t x)(t) \subset B\left(R\left([x]_{s}\right), \epsilon\right)$. Finally $\epsilon$ being arbitrary we obtain: $(P t x)(t) \subset R\left([x]_{t}\right)$.

\subsection{Optimal control problem for time-delay systems: the main result}

Now we state the main result on existence of optimal control for time-delay system. 
Theorem 5. Let $S(F, Q)$ be a time-delay control system. Assume the following hypothesis: (H2) The set valued map $F: G \times U \rightarrow C o m p R^{n}$ is continuous. (H3) The set valued map $Q: G \rightarrow$ Comp $R^{m}$ is upper semi-continuous. (H4) The set valued map $R: G \rightarrow C o m p R^{n}$ is convex values and verifying the relation: $R\left([x]_{t}\right) \subset \bar{B}\left(0, w\left(t,\|x\|_{t}\right)\right)$, for $t \in[0, T]$ where $w(t, y)$ verifies the hypothesis (H1). If a function $x \in C$ is a solution of $S(F, Q)$, then $x$ is a solution of the paratingent equation with delayed argument associate to $S(F, Q)$. And reciprocally, if the function $x \in C$ is a solution of the paratingent equation with delayed argument associate to $S(F, Q)$, then there exist a measurable function $u:[0, T] \rightarrow R^{m}$, such that $x$ is a solution of the $S(F, Q)$ and $u(t)$ the control corresponding to $x$.

Proof. i) Follow directly Theorem 2.

ii) Reciprocally, we have the following relations: $(P t x)(t) \subset R\left([x]_{t}\right)$ for $t \in[0, T]$ and $x(t)=$ $\zeta(t)$ for $t \in[\beta, 0]$. From Theorem 2 we obtained that $x$ is absolutely continuous on $[0, T]$ and $x^{\prime}(t) \in R\left([x]_{t}\right)=F\left([x]_{t}, Q\left([x]_{t}\right)\right.$ a.e on $[0, T]$. Moreover, since $x$ is continuous on $[0, T]$ and the map $R$ is upper semi-continuous on $G$ then $R$ is upper semi-continuous on $[0, T]$ and, according to the theorem of selector (see[4]), there exist a measurable function $u:[0, T] \rightarrow U$ such that $u(t) \in Q\left([x]_{t}\right)$ for $t \in[0, T]$. Otherwise there exist a control $u(t)$ corresponding to the solution $x(t)$ of $x^{\prime}(t) \in F\left([x]_{t}, u\right)$ for $t \in[0, T]$ and $x(t)=\zeta(t)$ for $t \in[\beta, 0]$.

Definition 6. Let $S(F, Q)$ be a time-delay control system, $M$ a compact subset of $C$ and $K$ an upper semi-continuous set valued map: $[0, T] \rightarrow C o m p R^{n}$. We say that the control $u(t)$ defined for $t \in\left[0, t_{1}\right]$, where $t_{1} \in[0, T]$, transfer $M$ to $K(t)$, if one of solutions $x(t)$ of the system $S(F, Q)$ corresponding to the control $u(t)$ verifies the following relations: $x \in M$ and $x\left(t_{1}\right) \in K\left(t_{1}\right)$.

Let $f\left([x]_{t}\right)$ be a real valued function defined on $G$ such that $\left|f\left([x]_{t}\right)\right|$ is majorated by a summable function for all $[x]_{t} \in G$. We consider the problem to find an optimal control $u(t)$ which transfers $M$ to $K(t)$ and which minimizes the following cost function: $J(x)=\int_{0}^{t} f\left([x]_{s}\right) d s$, where $x(t)$ is a solution of the time-delay system $S(F, Q)$ corresponding to control $u(t)$ and $t$ represents the first value such that $x(t) \in K(t)$.

Theorem 7. Let $S(F, Q)$ be a time-delay control system, verifying the hypothesis (H2), (H3) and (H4) of Theorem 3, $M$ a compact subset of $G$, and $K$ an upper semi-continuous set valued map: $[0, T] \rightarrow C o m p R^{n}$. If there exists at least one control which transfers $M$ to $K(t)$, then there exists an optimal control $u^{*}(t)$ (i.e. a measurable function $u^{*}(t)$ ) corresponding to the solution $x^{*}(t)$ and which verifies the following relations: $x^{*} \in M, x^{*}(t) \in K\left(t^{*}\right)$ for some $t^{*} \in[0, T]$, $\operatorname{Inf} J(x)=J\left(x^{*}\right)=\int_{0}^{t^{*}} f\left([x]_{s}\right) d s$ and $u^{*}(t) \in Q\left[x^{*}\right]_{t}$ a.e on $\left[0, t^{*}\right]$.

Proof. Consider the set $E_{0}(M, F)=\left\{x \in E(M, F)\right.$ such that $x\left(t_{1}\right) \in K\left(t_{1}\right)$ where $\left.t_{1} \in[0, T]\right\}$ and where $E(M, F)$ is the emission of the set $M$. We proof that $E_{0}(M, F)$ is compact in $E(M, F)$. Since $J(x)$ is a continuous function on the set $E_{0}(M, F)$ which is compact, then there exists a trajectory $x^{*}(t)$ for which $J(x)$ reaches its minimum on $E_{0}(M, F)$. According to Theorem 3, there exists a measurable function $u^{*}(t)$ such that: $x^{*}(t) \in F\left[x^{*}\right]_{t}$ a.e in $\left[t_{0}, t^{*}\right]$ and $u^{*}(t) \in Q\left[x^{*}\right]_{t}$ a.e in $\left[t_{0}, t^{*}\right]$. 


\section{Conclusion}

We have established the existence of the optimal control for a class of time-delay control systems by using an existence result of solution of paratingent equations with delayed argument and an equivalence theorem between the solutions of the paratingent equation with delayed argument and the time-delay control systems. Thus we have extended the class of the time-delay control systems which admit an optimal control.

\section{Acknowledgements}

The authors would like to thank an anonymous referee for his/her helpful suggestions for improving the original manuscript.

\section{References}

[1] J. P. Aubin, A. Cellina. Differential inclusions, Springer-Verlag, 1984.

[2] L. Boudjenah. Existence of the solutions of the paratingent equation with delayed argument. Electron. J. Diff. Eqns., 2005 (2005), No. 14, 1-8.

[3] S. Dadebo, R. Luus. Optimal control of time-delay systems by dynamic programming. Optim Control Appl. Methods, 13 (1992), No. 1, 29-41.

[4] M. Kisielewicz. Differential inclusions and optimal control. Kluwer, Dordrecht, The Netherlands, (1991).

[5] Y. Kuang. Delay differential equations with applications in population dynamics. Academic Press, Boston, (1993).

[6] W. Zygmunt. On some properties of a certain family of solutions of the paratingent equation. Ann. Univ. Marie Curie-Sklodowska, Lublin. Polonia. Sect. A, 28 (1976), 136-141. 